\title{
Recent advances in the frontline treatment of metastatic renal cell carcinoma
}

\author{
Camillo Porta ${ }^{1,2}$, Mimma Rizzo $^{3}$ \\ 'Department of Biomedical Sciences and Human Oncology, University of Bari 'A. Moro', Bari 70124, Italy. \\ 2Division of Medical Oncology, A.O.U. Consorziale Policlinico di Bari, Bari 70124, Italy. \\ ${ }^{3}$ Division of Translational Oncology, I.R.C.C.S. Istituti Clinici Scientifici Maugeri, Pavia 27100, Italy.
}

Correspondence to: Prof. Camillo Porta, Chair of Oncology, Department of Biomedical Sciences and Human Oncology, University of Bari 'A. Moro', and Division of Medical Oncology, A.O.U. Consorziale Policlinico di Bari, Piazza Giulio Cesare 11, 70124 Bari, Italy. E-mail: camillo.porta@gmail.com

How to cite this article: Porta C, Rizzo M. Recent advances in the frontline treatment of metastatic renal cell carcinoma. J Cancer Metastasis Treat 2021;7:49. https://dx.doi.org/10.20517/2394-4722.2021.86

Received: 1 Apr 2021 First Decision: 21 May 2021 Revised: 30 May 2021 Accepted: 9 Jun 2021 First online: 4 Jul 2021

Academic Editors: Lucio Miele, Hendrik P.A.M. Van-Poppel Copy Editor: Xi-Jun Chen Production Editor: Xi-Jun Chen

\begin{abstract}
We aim to describe the most recent advances in the upfront treatment of metastatic renal cell carcinoma, and to provide criteria - though often subjective - which could be used for treatment selection, by means of a critical review of the results of novel trials of immune-based combinations, coupled with personal considerations and experiences. To date, 5 immune-based combinations have been tested within large phase III trials; four of them yielded a significant overall survival benefit (Ipilimumab + Nivolumab, Pembrolizumab + Axitinib, Nivolumab + Cabozantinib and Pembrolizumab + Lenvatinib), while the combination of Avelumab + Axitinib, although reaching study primary endpoint, determined just a significant progression-free survival benefit. In terms of safety, the excess of adverse events is overall counterbalanced to the higher activity of the combinations. Overall, all the discussed immune-based combinations were ultimately approved by different regulatory authorities, and are indeed included in the most important international guidelines. Waiting for longer follow-ups and more mature trial data, as well as for real-world experiences, in the absence of validated biomarkers, our 1st line treatment choice cannot but rely on methodologically incorrect treatment comparisons, personal preferences, and experience.
\end{abstract}

Keywords: Renal cell carcinoma, immune checkpoint inhibitors, antiangiogenics, survival 


\section{INTRODUCTION}

In the past couple of years, we have witnessed a "third" revolution in the medical treatment of metastatic renal cell carcinoma (mRCC). After the development of targeted therapies and subsequently of single-agent immunotherapy, the outcome of our patients, affected by this once-orphan disease, has been further improved by the use of immune-based combinations, including either two different immune checkpoint inhibitors, or one vascular endothelial growth factor receptor-tyrosine kinase inhibitor (VEGFR-TKI) and one immune checkpoint inhibitor (ICI).

To date, five of these combinations have been tested within phase III trials; four of them yielded a significant overall survival (OS) benefit (Ipilimumab + Nivolumab, Pembrolizumab + Axitinib, Nivolumab + Cabozantinib, and Pembrolizumab + Lenvatinib ${ }^{[1-4]}$. While the combination of Avelumab + Axitinib reached the study primary endpoint, it only demonstrated a significant progression-free survival (PFS) benefit ${ }^{[5]}$. All these combinations were ultimately approved by different regulatory authorities, and are indeed included in the most important international guidelines ${ }^{[6-8]}$.

Another combination, Atezolizumab + Bevacizumab, although yielding formally positive results within another phase III study ${ }^{[9]}$, has not been registered, and its development in mRCC has been stopped due to conflicting results.

Here, we are going to review the results achieved by means of these novel combinations (with the exception of the Atezolizumab + Bevacizumab combination), and discuss how to practically select the best treatment choice for any new given patient entering our hospitals.

\section{METHODS}

We have extensively reviewed the literature regarding the use of immune-based combinations for the firstline treatment of mRCC, not only considering the primary publications of each trial, but also all available updates, either published in extenso or just presented at conferences.

\section{RESULTS}

As stated above, five immune-based combinations have been registered - by different regulatory authorities - for the upfront treatment of mRCC patients, thus yielding the status of novel standards of treatment in this specific setting. Four of the five combinations were able to significantly prolong OS, while the latter was able to improve PFS (but not OS).

Here, we summarize the results of the randomized, controlled, phase III trials of each of these five combinations, starting with those which achieved an OS benefit.

\section{Ipilimumab + Nivolumab (Checkmate-214)}

The very first randomized controlled phase III trial addressing the frontline role of an immune-based combination in mRCC patients was the Checkmate-214 study, which compared Ipilimumab + Nivolumab with Sunitinib for the first-line treatment of mRCC patients ${ }^{[1]}$.

In this study, 1096 patients were randomized to either the combination of the two ICIs, or Sunitinib monotherapy. Ipilimumab was administered intravenously (i.v.) at the dose of $1 \mathrm{mg} / \mathrm{kg}$ every 3 weeks for a total of four doses, while nivolumab was given at the dose of $3 \mathrm{mg} / \mathrm{kg}$ every 3 weeks, and continued also after the withdrawal of Ipilimumab. On the other hand, Sunitinib was administered, per os, at the 
conventional dose of $50 \mathrm{mg}$ once daily (o.d.), on a 4 weeks-on, 2 weeks-off, schedule.

Notably enough, the dose of the two ICIs were the opposite of those used in melanoma (i.e., Ipilimumab $3 \mathrm{mg} / \mathrm{kg}$ and Nivolumab $1 \mathrm{mg} / \mathrm{kg}$ ).

Co-primary, but hierarchical, endpoints were overall survival, followed by objective response rate (ORR), and finally by progression-free survival, among patients with International Metastatic RCC Database Consortium (IMDC) ${ }^{[10]}$ intermediate and poor risk features. However, good risk patients were also enrolled, representing an exploratory endpoint population.

In this first study ${ }^{[1]}$ with a median follow-up of 25.2 months, in the target population of intermediate and poor risk patients, the 18 -month OS rate was $75 \%$ [95\% confidence interval (CI): $70 \%-78 \%$ ] with the immune combination and 60\% (95\%CI: 55\%-65\%) with Sunitinib; however, the median OS was not reached with the immune combination vs. 26.0 months with Sunitinib [hazard ratio $(\mathrm{HR})=0.63 ; P<0.001$ ]. The ORR was $42 \%$ with immune combination $v s .27 \%$ with Sunitinib $(P<0.001)$, with a huge percentage of patients from the combination arm achieving a complete response, $9 \%$ ( $v s .1 \%$ for Sunitinib-treated patients). Finally, median PFS was 11.6 months and 8.4 months for immune combination and Sunitinib, respectively ( $\mathrm{HR}=0.82 ; P=0.03$ ), a difference that was not statistically significant per the prespecified 0.009 threshold.

As far as safety, treatment-related adverse events (TRAEs) occurred in $93 \%$ of the subjects randomized to receive Ipilimumab + Nivolumab, and in $97 \%$ of Sunitinib-treated patients, with grade 3 or 4 events occurring in $46 \%$ and $63 \%$ of the patients, respectively. Finally, TRAEs leading to treatment discontinuation occurred in $22 \%$ and $12 \%$ of the patients, respectively.

Subsequent analyses, conducted at different follow-up times, confirmed the above results ${ }^{[1-13]}$; indeed, at the latest study update ${ }^{[13]}$, after 4 years minimum follow-up, the immune combination yielded better results in intermediate and poor risk patients, both in terms of OS (median, 48.1 months vs. 26.6 months; $\mathrm{HR}=0.65$; 95\%CI: 0.54-0.78) and of ORR ( $39.1 \%$ vs. 32.4\%) while, as often happens with ICIs, PFS benefit proved to be limited, realistically due to their latency of action, time being needed for the activation, and expansion and trafficking of the effector immune cells.

On the contrary, in favorable risk patients, the difference in OS was not statistically significant $(\mathrm{HR}=0.93$; 95\%CI: $0.62-1.4$ ), median OS having not been reached in either arm, and OS probabilities at 4 years being similar between the two treatment arms, $65.1 \%$ for the immune combination $v$ s. $68.9 \%$ for Sunitinib ${ }^{[13]}$.

\section{Pembrolizumab + Axitinib (Keynote-426)}

In this study, 1062 previously untreated mRCC patients were randomized to either the combination of the anti-PD1 monoclonal antibody Pembrolizumab with the highly selective VEGFRs inhibitor Axitinib, or Sunitinib monotherapy ${ }^{[2]}$.

Pembrolizumab was administered i.v. at a dose of $200 \mathrm{mg}$ every 3 weeks for a maximum of 35 administrations, while Axitinib was administered orally at a dose of $5 \mathrm{mg}$ twice daily (t.i.d.) until disease progression or intolerance; Axitinib dose could be increased till a maximum of $10 \mathrm{mg}$ t.i.d., in case of adequate tolerance, or reduced till $2 \mathrm{mg}$ t.i.d. in case of safety concerns. Sunitinib was administered orally at a dose of $50 \mathrm{mg}$ o.d., on the classical 4:2 schedule, but starting from the second cycle dose reductions till a minimum of $25 \mathrm{mg}$ o.d. were allowed in case of toxicity. Finally, in the case of confirmed complete 
response, patients could discontinue treatment.

The dual primary end points were OS and PFS according to blinded, independent central review, while key secondary end points were ORR, duration of response, and safety.

In this first report ${ }^{[2]}$ with a median follow-up of 12.8 months, the median OS was not reached in either group, the estimated percentage of patients who were alive at 12 months was $89.9 \%$ (95\%CI: 86.4-92.4) in the combination arm, and $78.3 \%$ (95\%CI: 73.8-82.1) in the monotherapy arm.

Median PFS was 15.1 months (95\%CI: 12.6-17.7) in the combination arm and 11.1 months (95\%CI: 8.712.5 ) in the control arm; the HR for disease progression or death was 0.69 (95\%CI: $0.57-0.84 ; P<0.001$ ).

The above survival benefits of the immune-based combination were observed in all the three IMDC risk categories.

ORR was 59.3\% (95\%CI: 54.5-63.9) in the immune-based combination group, where $5.8 \%$ of patients experienced a complete response, and $35.7 \%(95 \% \mathrm{CI}: 31.1-40.4)$ in the monotherapy group $(P<0.001)$, where just $1.9 \%$ of the patients had a complete response.

As far as safety, adverse events of any cause occurred in $98.4 \%$ of the patients randomized to receive the combination, and in $99.5 \%$ of Sunitinib-treated patients, with grade 3 or higher TRAEs occurring in $62.9 \%$ and $58.1 \%$ of the patients, respectively.

Finally, in the Pembrolizumab + Axitinib group, adverse events of any cause leading to discontinuation of either drug, discontinuation of both drugs, interruption of either drug, or dose reduction of Axitinib occurred in $30.5 \%, 10.7 \%, 69.9 \%$, and $20.3 \%$ of patients, respectively; on the contrary, in the Sunitinib group, adverse events of any cause led to discontinuation in $13.9 \%$ of patients, interruption in $49.9 \%$, and dose reduction in $30.1 \%$.

At the second study update ${ }^{[14]}$, at a median follow-up of 30.6 months, the immune-based combination continued to yield superior clinical outcomes over Sunitinib. Indeed, the estimated OS rate at 24 months was $74.4 \%$ (95\%CI: 69.9-78.2) in the Pembrolizumab + Axitinib group and 65.5\% (95\%CI: 60.8-69.8) in the Sunitinib group. In IMDC good risk patients, however, 12 and 24 months OS rates were $95.6 \%$ (95\%CI: 90.5-98.0) and $85.3 \%$ (95\%CI: 78.1-90.2) for the combination therapy, and 94.6\% (95\%CI: 89.0-97.4) and 87.7\% (95\%CI: 80.6-92.2), clearly indicating no added benefit from the combination, as compared to monotherapy, in this subgroup of patients.

Median PFS was 15.4 months (95\%CI: 12.7-18.9) with the combination, vs. 11.1 months (95\%CI: 9.1-12.5) with Sunitinib monotherapy $(\mathrm{HR}=0.71 ; 95 \% \mathrm{CI}$ : 0.60-0.84; $P<0.0001)$.

ORR was 60\% (95\%CI: 55.4-64.8) with Pembrolizumab + Axitinib, compared with 40\% (95\%CI: 35.2-44.7) with Sunitinib $(P<0.0001) ; 38(9 \%)$ of 432 patients in the combination arm and $13(3 \%)$ of 429 patients in the monotherapy arm had a complete response ${ }^{[14]}$.

Finally, neither new safety signals, nor new treatment-related deaths, were observed with a longer follow-up, compared with the first study report. 
As a whole, the results of the Keynote-426 trial support the combination of Pembrolizumab + Axitinib as another standard of care for mRCC patients, irrespective of IMDC risk groups (although with a limited added benefit for good risk patients).

\section{Nivolumab + Cabozantinib (Checkmate 9ER)}

In this study, 651 previously untreated mRCC patients were randomized to either the combination of the anti-PD 1 monoclonal antibody Nivolumab with the multikinase inhibitor Cabozantinib, or Sunitinib monotherapy ${ }^{[3]}$.

The doses of the three agents were the following: $240 \mathrm{mg}$ i.v. every 2 weeks for Nivolumab, $40 \mathrm{mg}$ o.d. per os (i.e., one dose level less than the starting monotherapy dose) for Cabozantinib, and $50 \mathrm{mg}$ o.d. per os, 4 weeks on, two weeks off, for Sunitinib. Dose reductions were not allowed for Nivolumab, but permitted for the two oral agents.

Treatment continued until disease progression or unacceptable toxicity occurred, with a maximum 2-year duration for Nivolumab.

The primary end point was PFS among the intention-to-treat population, while secondary end points were OS, ORR, time to and duration of response, as well as safety.

At a median follow-up of 18.1 months, median PFS was 16.6 months (95\%CI: 12.5-24.9) with the immunebased combination, and 8.3 months (95\%CI: 7.0-9.7) with Sunitinib, a difference that was statistically significant, yielding a reduction in the risk of progression or death of $49 \%(\mathrm{HR}=0.51$; 95\%CI: 0.41-0.64; $P<$ 0.001 ). Furthermore, Nivolumab + Cabozantinib also yielded a significant OS benefit over Sunitinib, the 12month OS probability being $85.7 \%$ (95\%CI: 81.3-89.1) with the combination, and 75.6\% (95\%CI: 70.5-80.0) with the monotherapy. Overall, the reduction in the risk of death was $40 \%$ ( $\mathrm{HR}=0.60$; 98.89\%CI: 0.40-0.89; $P=0.001)$, though at the time of publication median OS was not reached in either group.

ORR was 55.7\% (95\%CI: 50.1-61.2) with Nivolumab + Cabozantinib, and 27.1\% (95\%CI: 22.4-32.3) with Sunitinib $(P<0.001)$, while complete response rate was $8.0 \%$ for the immune-based combination and $4.6 \%$ for the monotherapy ${ }^{[3]}$.

PFS benefit of the combination over Sunitinib was consistent across the three IMDC risk groups: $\mathrm{HR}=0.62$ (95\%CI: 0.38-1.01), HR = 0.54 (95\%CI: 0.40-0.72), and HR = 0.37 (95\%CI: 0.23-0.58) for good, intermediate, and poor-risk patients, respectively. The same did not occur when OS was taken into consideration: $\mathrm{HR}=$ 0.84 (95\%CI: $0.35-1.97), \mathrm{HR}=0.70$ (95\%CI: 0.46-1.07) and $\mathrm{HR}=0.37$ (95\%CI: $0.21-0.66)$ for each of the three groups.

TRAEs of any grade and TRAEs grade 3 or higher were reported in $96.6 \%$ and $60.6 \%$ of the patients treated with Nivolumab + Cabozantinib (vs. 93.1\% and 50.9\% in those treated with Sunitinib), respectively. The percentage of adverse events of any cause leading to the discontinuation of a trial drug were $19.7 \%$ with the combination (6.6\% discontinued Nivolumab only, 7.5\% Cabozantinib only, and 5.6\% both agents), and $16.9 \%$ with Sunitinib. Finally, patients reported better health-related quality of life with Nivolumab + Cabozantinib than with Sunitinib. 
Study results have been recently updated at the 2021 Genitourinary Cancers Symposium ${ }^{[15]}$.

Thus, the combination of Nivolumab + Cabozantinib was added to the previous standards of care for mRCC patients, irrespective of IMDC risk groups (although a limited OS benefit for good risk patients was showed).

\section{Pembrolizumab + Lenvatinib (CLEAR study)}

In this study, 1069 previosuly untreated mRCC patients were randomly assigned to receive Pembrolizumab + Lenvatinib, Lenvatinib + Everolimus, or Sunitinib ${ }^{[4]}$.

In the Pembrolizumab + Lenvatinib group, the anti-PD1 monoclonal antibody and the multikinase inhibitor were administered at the dose of $200 \mathrm{mg}$ i.v., every 21 days, and $20 \mathrm{mg}$ per os, on a continuous dosing, respectively.

In the Lenvatinib + Everolimus group, Lenvatinib and Everolimus were administered at the dose of $18 \mathrm{mg}$ and $5 \mathrm{mg}$ o.d. per os, while Sunitinib was administered at the dose of $50 \mathrm{mg}$ orally o.d. on the classical 4:2 schedule.

The primary end point was PFS, secondary endpoints being OS, ORR, and safety.

At a median follow-up of 26.6 months, median PFS as determined by independent review was significantly longer in the Pembrolizumab + Lenvatinib arm than in the Sunitinib arm: 23.9 months (95\%CI: 20.8-27.7) vs. 9.2 months (95\%CI: 6.0-11.0), leading to a HR of 0.39 (95\%CI: 0.32-0.49; $P<0.001$ ). Furthermore, median PFS was also significantly longer in the Lenvatinib + Everolimus than in the Sunitinib arm: 14.7 months (95\%CI: 11.1-16.7) vs. 9.2 months (95\%CI: 6.0-11.0), leading to a HR of 0.65 (95\%CI: 0.53-0.80; $P<$ $0.001)$.

In the OS analysis, $79.2 \%$ of the patients treated with the immune-based combination, $66.1 \%$ of the patients in the non-ICI-containing combination, and $70.4 \%$ of the patients in the Sunitinib group were alive at 24 months, although median OS was not reached with any of the three treatments.

OS was significantly longer with Pembrolizumab + Lenvatinib than with Sunitinib (HR $=0.66$; 95\%CI: 0.49$0.88, P=0.005$ ), while it was not the case when the combination of Lenvatinib + Everolimus was compared with Sunitinib (HR $=1.15$; 95\%CI: 0.88-1.50; $P=0.30)$.

ORR was $71.0 \%$ with Pembrolizumab + Lenvatinib, 53.5\% with Lenvatinib + Everolimus, and $36.1 \%$ with Sunitinib. The relative risk of mortality with the immune-based combination $v s$. Sunitinib was thus 1.97 (95\%CI: 1.69-2.29), while it was 1.48 (95\%CI: 1.26-1.74) for the Lenvatinib + Everolimus combination $v s$. Sunitinib. The percentage of patients achieving a complete response was $16.1 \%$ in the Pembrolizumab + Everolimus arm, $9.8 \%$ in the Lenvatinib + Everolimus arm, and finally $4.2 \%$ in the Sunitinib arm.

Almost all patients in each group had adverse events of any cause: $99.7 \%$ in both the Pembrolizumab + Lenvatinib and Lenvatinib + Everolimus arm, and 98.5\% in the Sunitinib arm. Grade 3 or higher adverse events of any cause occurred in $82.4 \%$ of the patients who received Pembrolizumab + Lenvatinib, in $83.1 \%$ of the patients who received Lenvatinib + Everolimus, and in $71.8 \%$ of the patients who received Sunitinib. In the ICI-containing combination, adverse events of any grade led to discontinuation of Pembrolizumab, Lenvatinib, or both agents in $37.2 \%$ of the treated cases (Pembrolizumab: $28.7 \%$, Lenvatinib: $25.6 \%$, and both 
drugs: $13.4 \%$ ), to dose reduction of Lenvatinib in $68.8 \%$ of patients, and to interruption of Lenvatinib, Pembrolizumab, or both drugs in $78.4 \%$ of patients.

In the Lenvatinib + Everolimus arm, adverse events of any grade led to discontinuation of Lenvatinib, Everolimus, or both agents in $27.0 \%$ of patients (Lenvatinib: $22.0 \%$, Everolimus: $24.8 \%$, and both drugs: $18.9 \%$ ), to dose reduction of Lenvatinib, Everolimus, or both agents in $73.2 \%$ of cases, and led to interruption of Lenvatinib, Everolimus, or both drugs in $83.4 \%$ of the patients.

Finally, in the Sunitinib arm, the relative figures were: $14.4 \%$ for discontinuation, 50.3\% for dose reduction, and $53.8 \%$ for treatment interruption.

The combination of Pembrolizumab + Lenvatinib thus joined the other three above mentioned combination as possible standards of care for the treatment of mRCC patients.

\section{Avelumab + Axitinib (JAVELIN Renal 101)}

In this study, 886 previously untreated mRCC patients were randomized to either the combination of the anti-PD-L1 monoclonal antibody Avelumab with the VEGFR-TKI Axitinib, or Sunitinib monotherapy ${ }^{[5]}$.

Avelumab was administered at a dose of $10 \mathrm{mg} / \mathrm{kg}$ of body weight as a $1 \mathrm{~h}$ intravenous infusion every 2 weeks, following premedication with an antihistamine and acetaminophen. Axitinib was administered orally at a starting dose of $5 \mathrm{mg}$ twice daily on a continuous dosing, while Sunitinib was administered at the dose of $50 \mathrm{mg}$ orally, o.d., on a 4 weeks-on, 2 weeks-off, schedule. Dose escalations and reductions for Axitinib, and dose reductions for Sunitinib, were allowed per protocol.

Although the original primary objective of the study was PFS in all enrolled patients, during study conduction, an amendment made PFS and OS among patients with PD-L1-positive tumors the study's novel co-primary endpoint, with key secondary end points being PFS in the overall population, ORR, and safety.

Among the patients who had PD-L1-positive tumors (560, 63.2\%), PFS was significantly longer among those who received the immune-based combination, as compared to those who received Sunitinib monotherapy. Indeed, the median PFS was 13.8 months (95\%CI: 11.1-NE) with Avelumab + Axitinib, as compared with 7.2 months (95\%CI: 5.7-9.7) with Sunitinib (stratified HR $=0.61$; 95\%CI: 0.47-0.79; $P<0.001$ ).

As far as OS, among PD-L1-positive patients, deaths from any cause were observed in 37 patients (13.7\%) who received the combination $v s .44$ (15.2\%) who received Sunitinib (stratified HR =0.82; 95\%CI: 0.53-1.28; $P=0.38)$.

Among the patients with PD-L1-positive tumors, the confirmed ORR was 55.2\% (95\%CI: 49.0-61.2) with Avelumab + Axitinib vs. $25.5 \%$ (95\%CI: 20.6-30.9) with Sunitinib, confirmed complete response rates being $4.4 \%$ and $2.1 \%$, respectively.

In the overall population, median PFS was also significantly longer with Avelumab + Axitinib than with Sunitinib: 13.8 months (95\%CI: 11.1-NE) vs. 8.4 months (95\%CI: 6.9-11.1), with HR for progression or death being 0.69 (95\%CI: $0.56-0.84 ; P<0.001)$. 
As far as OS, no statistically significant difference was evidenced. Indeed, deaths from any cause were recorded in 63 patients $(14.3 \%)$ who received the combination vs. 75 patients $(16.9 \%)$ who received Sunitinib monotherapy $(\mathrm{HR}=0.78 ; 95 \% \mathrm{CI}: 0.55-1.08 ; P=0.14)$.

A subgroup analysis showed a significant PFS benefit for the immune-based combination across the three IMDC risk categories: indeed, HR for progression or death was 0.50 (0.26-0.97), 0.64 (0.47-0.88), and 0.53 (0.30-0.93) for good-, intermediate-, and poor-risk patients, respectively.

As far as safety, adverse events of any grade occurred in $99.5 \%$ and $99.3 \%$ of patients who received the immune-based combination and Sunitinib, respectively; adverse events of grade 3 or higher during treatment occurred $71.2 \%$ and $71.5 \%$ of the patients in the respective groups. Furthermore, adverse events leading to discontinuation of both Avelumab and Axitinib or of single-agent Sunitinib were 7.6\% and 13.4\%, respectively.

A recent publication updated the results of the JAVELIN Renal 101 trial, after a minimum follow-up of 13 month $s^{[16]}$. Again, PFS was significantly longer in those patients with PD1-positive tumors treated with the combination, as compared to monotherapy arm (13.8 months vs. 7.0 months; HR $=0.62$; 95\%CI: 0.490$0.777 ; P<0.0001)$. In the overall patient population, median PFS was 13.3 months for the combination, and 8.0 months for Sunitinib (HR $=0.69$; 95\%CI: 0.574-0.825; $P<0.0001$ ). HR for OS was 0.828 (95\%CI: 0.596$1.151 ; P=0.1301)$ for the PD-L1-positive population, and of 0.796 (95\%CI: $0.616-1.027 ; P=0.0392)$ for the overall population.

As a whole, the results of the four trial which yielded an OS benefit are summarized in Table 1; results from JAVELIN Renal 101 were not included in the table, due to the lack of OS benefit, which makes - in our opinion - the Avelumab + Axitinib option less intriguing.

\section{DISCUSSION}

Given all the data above presented, how can we navigate between them? Or, in other words, how can we make treatment choices in real world?

First of all, we should avoid making direct comparisons between studies which are extremely different in terms of: agents used, study endpoints, primary efficacy patient populations, different follow-up (meaning that there are still many immature data), distribution of patients between the three IMDC prognostic groups, and, as a consequence, also results.

Despite all the above, it is almost impossible not to do some general considerations.

First, the Pembrolizumab + Lenvatinib combination seems to be the more potent, having achieved astounding PFS benefit, the largest ever observed in mRCC, together with an unprecedented disease control rate $(90 \%)$ and percentage of complete responses $(16 \%)^{[4]}$. However, we should beware of patient selection. Indeed, among the discussed trials, CLEAR ${ }^{[4]}$ has enrolled the lower amount of IMDC poor risk patients (9\%) and, together with Checkmate $214^{[1]}$, the larger amount of intermediate risk patients (60\%).

A high treatment activity has also been observed in Keynote 426 (with almost the same issue relative to patient selection as in CLEAR $)^{[2]}$, as well as in Checkmate $9 \mathrm{ER}^{[3]}$, where there is probably a better balance in terms of patient selection. 
Table 1. Cross-trial comparison of first line immune-based combinations for the treatment of metastatic renal cell carcinoma, according to the latest update of each trial. Only studies yielding an OS benefit are summarized here

\begin{tabular}{|c|c|c|c|c|c|c|c|c|}
\hline Study & \multicolumn{2}{|c|}{ Checkmate $214^{[1,11,12,13]}$} & \multicolumn{2}{|c|}{ Keynote $426^{[2,15]}$} & \multicolumn{2}{|c|}{ Checkmate 9ER ${ }^{[3,16]}$} & \multicolumn{2}{|c|}{ CLEAR $^{[4]}$} \\
\hline Tx arms & \multicolumn{2}{|c|}{$\begin{array}{l}\text { Ipilimumab + Nivolumab } \\
\text { vs. Sunitinib }\end{array}$} & \multicolumn{2}{|c|}{$\begin{array}{l}\text { Pembrolizumab + Axitinib } \\
\text { vs. Sunitinib }\end{array}$} & \multicolumn{2}{|c|}{$\begin{array}{l}\text { Nivolumab + Cabozantinib } \\
\text { vs. Sunitinib }\end{array}$} & \multicolumn{2}{|c|}{$\begin{array}{l}\text { Pembrolizumab + Lenvatinib } \\
\text { vs. Sunitinib }\end{array}$} \\
\hline Prognostic groups & \multicolumn{2}{|c|}{$\begin{array}{l}\text { Good } 23 \% \text {, intermediate } \\
61 \% \text {, poor } 17 \%\end{array}$} & \multicolumn{2}{|c|}{$\begin{array}{l}\text { Good } 32 \% \text {, intermediate } \\
55 \% \text {, poor } 13 \%\end{array}$} & \multicolumn{2}{|c|}{$\begin{array}{l}\text { Good } 23 \% \text {, intermediate } \\
58 \% \text {, poor } 19 \%\end{array}$} & \multicolumn{2}{|c|}{$\begin{array}{l}\text { Good } 31 \% \text {, intermediate } \\
60 \% \text {, poor } 9 \%\end{array}$} \\
\hline Target population & \multicolumn{2}{|c|}{$\begin{array}{l}\text { Intermediate \& Poor risk } \\
\text { patients }\end{array}$} & \multicolumn{2}{|c|}{ All risk groups } & \multicolumn{2}{|c|}{ All risk groups } & \multicolumn{2}{|c|}{ All risk groups } \\
\hline Follow-up, months & \multicolumn{2}{|l|}{55} & \multicolumn{2}{|l|}{30.6} & \multicolumn{2}{|l|}{23.5} & \multicolumn{2}{|l|}{27} \\
\hline ORR, \% & 42 & 27 & 60 & 40 & 55 & 28 & 71 & 36 \\
\hline CR & 10 & 1 & 9 & 3 & 9 & 4 & 16 & 4 \\
\hline PR & 32 & 25 & 51 & 37 & 46 & 24 & 55 & 32 \\
\hline SD & 31 & 44 & 23 & 35 & 33 & 42 & 19 & 38 \\
\hline DCR & 73 & 70 & 83 & 75 & 88 & 70 & 90 & 74 \\
\hline PD & 19 & 17 & 11 & 17 & 6 & 14 & 5 & 14 \\
\hline Median OS, months & 48.1 & 26.6 & NE & 35.7 & NE & 29.5 & NE & NE \\
\hline$(95 \% \mathrm{Cl})$ & (35.6-NE) & $(22.1-33.5)$ & & (33.3-NE) & & (28.4-NE) & (33.6- & \\
\hline HR for OS $(95 \% \mathrm{Cl})$ & \multicolumn{2}{|c|}{$0.65(0.54-0.88)$} & \multicolumn{2}{|c|}{$0.68(0.55-0.85)$} & \multicolumn{2}{|c|}{$0.66(0.50-0.87)$} & \multicolumn{2}{|c|}{$0.66(0.49-0.88)$} \\
\hline $\begin{array}{l}\text { Reduction in the risk of } \\
\text { death, \% }\end{array}$ & \multicolumn{2}{|l|}{35} & \multicolumn{2}{|c|}{32} & \multicolumn{2}{|l|}{34} & \multicolumn{2}{|l|}{34} \\
\hline Median PFS, months & 11.2 & 8.3 & 15.4 & 11.1 & 17.0 & 8.3 & 23.9 & 9.2 \\
\hline HR for PFS $(95 \% \mathrm{Cl})$ & \multicolumn{2}{|c|}{$0.74(0.62-0.88)$} & \multicolumn{2}{|c|}{$0.71(0.60-0.84)$} & \multicolumn{2}{|c|}{$0.52(0.43-0.64)$} & \multicolumn{2}{|c|}{$0.39(0.32-0.49)$} \\
\hline $\begin{array}{l}\text { Reduction in the risk of } \\
\text { PD or death, \% }\end{array}$ & \multicolumn{2}{|l|}{26} & \multicolumn{2}{|l|}{29} & \multicolumn{2}{|l|}{48} & \multicolumn{2}{|l|}{61} \\
\hline
\end{tabular}

"Third arm of CLEAR study (Lenvatinib + Everolimus) not included in this summary table. Tx: Treatment; ORR: overall response rate; CR: complete response; PR: partial response; SD: stable disease; DCR: disease control rate; PD: progressive disease; OS: overall survival; NE: not established (yet); HR: hazard ratio; Cl: confidence interval; PFS: progression-free survival.

In terms of OS, still the "Holy Grail" of oncology, a similar reduction in the risk of death was recorded across all trials $(35 \%, 32 \%, 34 \%$, and $34 \%$ for Checkmate 214 , Keynote 426 , Checkmate 9ER, and CLEAR, respectively): however, we should also acknowledge that OS data in CLEAR are not completely mature, a huge amount of censoring being evident after the 12-month mark ${ }^{[4]}$; furthermore, the HR for OS increased from the second interim analysis to the third one (i.e., from 0.47 to 0.66 ), although realistically due to the effect of the use of post-progression treatments in the control $\mathrm{arm}^{[4]}$.

As far as the all-immune combination tested within Checkmate 214, it is important to highlight the patient selection made a priori. The study, although enrolling all newcomers, was indeed designed to demonstrate efficacy in IMDC intermediate and poor-risk patients ${ }^{[1]}$.

Some interesting considerations may also come from the analysis of the shapes of the curves of these trials, particularly important when immunotherapy is concerned.

Taking a look at the PFS curves of Checkmate 214, it is clear that no separation of the curves occurs for the first months ${ }^{[1]}$, as shown in Figure 1; as a combination of two ICIs, Ipilimumab + Nivolumab requires time to activate and expand the effector populations of the immune system, and thus the heads of the curves separate later compared with what is observed with the combination of an ICI and a VEGFR-TKI. At least theoretically, this means that, in the case of a very aggressive tumor, we risk losing a certain number of patients during the first months of treatment. 
Checkmate 214

Keynote 426

Checkmate 9ER

CLEAR
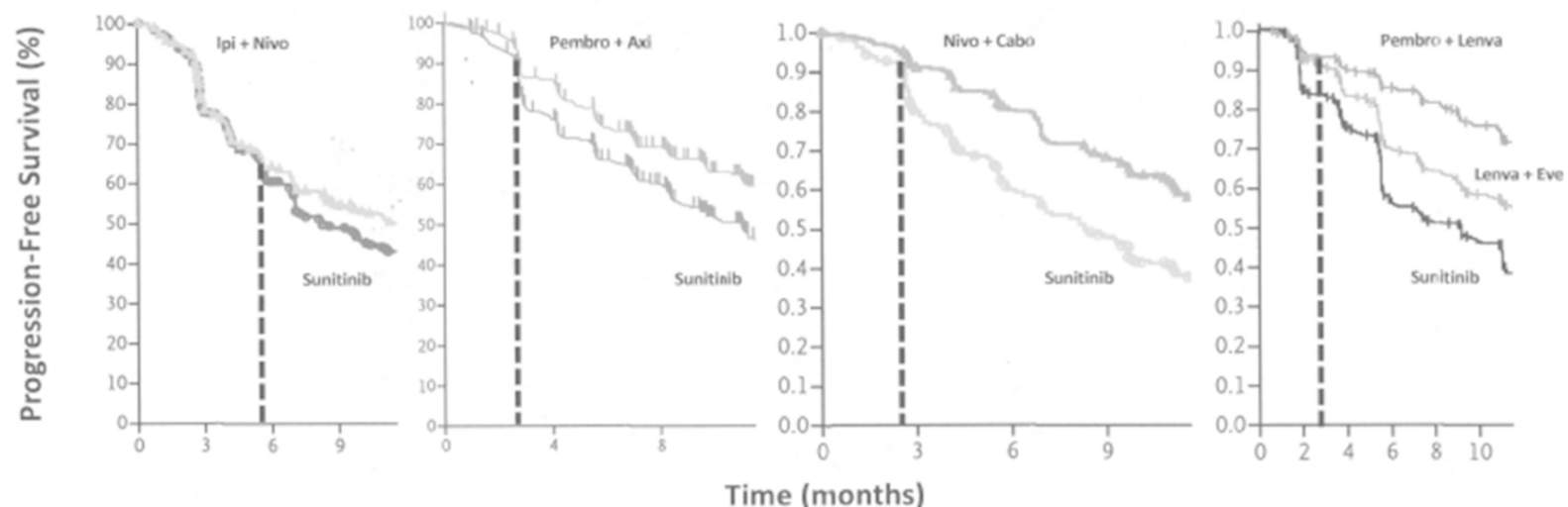

Time (months)

Figure 1. Comparison between the heads of the PFS curves from Checkmate 214, Keynote 426, Checkmate 9ER, and CLEAR. Possible interpretation is reported within the text (modified from ${ }^{[1-4]}$ ).

After these initial months, separation of the curves, and consequently, a positive impact on median survival occurs; thus, there is no surprise in the observation that four out of five of the considered combinations were able to improve OS over the same control treatment (i.e., Sunitinib).

The lack of OS impact of the Avelumab-based combination might be related to a lower activity/efficacy of anti-PD-L1 agents, as compared to anti-PD1 (at least in mRCC), as also suggested by the results of the IMmotion 151 trial of Atezolizumab + Bevacizumab ${ }^{[9]}$. Indeed, it is clear that not all ICIs are born equal ${ }^{[17]}$, although these differences may also be dependent on specific tumor types.

Finally, with immunotherapy, a survival plateau is often observed after few years, suggesting the presence of a certain number of long-survivors ("cured") patients. For sure, this tail looks striking in Checkmate $214^{[1,11-13]}$, although follow-up periods across the different studies are too different to draw definitive conclusions. Furthermore, it is definitely too early to confirm the presence of cured patients by any of the tested immune-based combos.

As a whole, the only possible, though highly empiric, present driver of our therapeutical choice could be the biological aggressiveness of the tumor. Indeed, in the case of a very aggressive disease, the use of an ICI plus a VEGFR-TKI seems a very reasonable choice, in order to try to control early disease growth, while waiting for the "tail" effect of immunotherapy.

Otherwise, one could head for the long-term benefit of the immune combination, trying to spare the additional toxicities deriving from the continuous use of the VEGFR-TKI.

In conclusion, with the present lack of biomarkers predictive of response from (or, on the contrary, of inefficacy of) the different available treatments ${ }^{[18]}$, we should still rely just on clinical parameters (like those included in the IMDC risk classification system), personal judgement, and experience.

As far as safety, it is usually considered another key element for choosing the best treatment for each given patient. In the recent past, Bracarda et al. ${ }^{[19]}$ proposed that the polarizing toxicity of each treatment (i.e., the 
most frequent toxicity) should be used to create a new algorithm able to identify those patients unquestionably unsuitable for each therapy, across different treatment lines.

Although a useful practical suggestion, this approach is now flawed by the fact that the safety profiles of combinations of an ICI plus a VEGFR-TKI are extremely similar, not to take into account that the trade-off between efficacy and safety a first line patient is willing to accept is usually unbalanced in favor of efficacy.

Furthermore, beyond the number and severity of TRAE, we should also consider their relative duration. Indeed, multiple, long-lasting, low-grade, adverse events usually negatively impact on patients' quality of life more than single, high-grade, toxicities, especially if rapidly resolving; not to take into account that, for patients treated with the Ipilimumab + Nivolumab immune combination, severe adverse events are mainly limited to the induction phase, when both ICIs are simultaneously given, while they tend to be less troublesome during the Nivolumab-alone maintenance phase of the treatment.

In conclusion, waiting for more mature trial data, as well as for real-world experiences, in the absence of validated biomarkers, our first line treatment choice cannot but rely on methodologically incorrect treatment comparisons, personal preferences, and experience.

\section{DECLARATIONS}

Authors' contributions

Made equal contribution in the collection and critical interpretation of the data summarized in this manuscript: Porta C, Rizzo M

\section{Availability of data and materials}

Not applicable.

\section{Financial support and sponsorship}

None.

\section{Conflicts of interest}

Porta C acted as a Consultant and/or Speaker for Angelini, Astra Zeneca, BMS, Eisai, EUSA, General Electric, Ipsen, Janssen, Merck, MSD, Novartis and Pfizer; as an Expert Testimony for EUSA and Pfizer; and as a Protocol Steering Committee Member for BMS, Eisai and EUSA; finally, he received travel support from Roche. Rizzo M acted as a Consultant and/or Speaker for Novartis, Pfizer, and MSD.

\section{Ethical approval and consent to participate}

Not applicable.

\section{Consent for publication}

Not applicable.

\section{Copyright}

(c) The Author(s) 2021.

\section{REFERENCES}

1. Motzer RJ, Tannir NM, McDermott DF, et al. Nivolumab plus Ipilimumab versus Sunitinib in Advanced Renal-Cell Carcinoma. $N$ Engl J Med 2018;378:1277-90. DOI PubMed PMC

2. Rini BI, Plimack ER, Stus V, et al. Pembrolizumab plus Axitinib versus Sunitinib for advanced renal-cell carcinoma. $N$ Engl $J$ Med 2019;380:1116-27. DOI PubMed

3. Choueiri TK, Powles T, Burotto M, et al. Nivolumab plus Cabozantinib versus Sunitinib for advanced renal-cell carcinoma. $N$ Engl $J$ 
Med 2021;384:829-41. DOI PubMed

4. Motzer RJ, Alekseev B, Rha SY, et al. Lenvatinib plus Pembrolizumab or Everolimus for advanced renal cell carcinoma. $N$ Engl J Med 2021;384:1289-300. DOI PubMed

5. Motzer RJ, Penkov K, Haanen J, et al. Avelumab plus axitinib versus sunitinib for advanced renal-cell carcinoma. $N$ Engl J Med 2019;380:1103-15. DOI PubMed PMC

6. ESMO Guidelines Committee. eUpdate - renal cell carcinoma treatment recommendations https://www.esmo.org/guidelines/genitourinary-cancers/renal-cell-carcinoma/eupdate-renal-cell-carcinoma-treatmentrecommendations-3 [Last accessed on 8 Jul 2021].

7. Albiges L, Powles T, Staehler M, et al. Updated European Association of Urology guidelines on renal cell carcinoma: immune checkpoint inhibition is the new backbone in first-line treatment of metastatic clear-cell renal cell carcinoma. Eur Urol 2019;76:151-6. DOI PubMed

8. NCCN Clinical Practice Guidelines in Oncology (NCCN Guidelines $\left.{ }^{\circledR}\right)$. Kidney Cancer, Version 3.2021. Available from: https://www.nccn.org/professionals/physician_gls/pdf/kidney.pdf. [Last accessed on 12 Aug 2021].

9. Rini BI, Powles T, Atkins MB, et al. Atezolizumab plus bevacizumab versus sunitinib in patients with previously untreated metastatic renal cell carcinoma (IMmotion151): a multicentre, open-label, phase 3, randomised controlled trial. Lancet 2019;393:2404-15. DOI PubMed

10. Heng DY, Xie W, Regan MM, et al. External validation and comparison with other models of the International Metastatic Renal-Cell Carcinoma Database Consortium prognostic model: a population-based study. Lancet Oncol 2013;14:141-8. DOI PubMed PMC

11. Motzer RJ, Rini BI, McDermott DF, et al. Nivolumab plus ipilimumab versus sunitinib in first-line treatment for advanced renal cell carcinoma: extended follow-up of efficacy and safety results from a randomised, controlled, phase 3 trial. Lancet Oncol 2019;20:137085. DOI PubMed PMC

12. Motzer RJ, Escudier B, McDermott DF, et al. Survival outcomes and independent response assessment with nivolumab plus ipilimumab versus sunitinib in patients with advanced renal cell carcinoma: 42-month follow-up of a randomized phase 3 clinical trial. J Immunother Cancer 2020;8:e000891. DOI PubMed PMC

13. Albiges L, Tannir NM, Burotto M, et al. Nivolumab plus ipilimumab versus sunitinib for first-line treatment of advanced renal cell carcinoma: extended 4-year follow-up of the phase III CheckMate 214 trial. ESMO Open 2020;5:e001079. DOI PubMed PMC

14. Powles T, Plimack ER, Soulières D, et al. Pembrolizumab plus Axitinib versus Sunitinib monotherapy as first-line treatment of advanced renal cell carcinoma (KEYNOTE-426): extended follow-up from a randomised, open-label, phase 3 trial. Lancet Oncol 2020;21:1563-73. DOI PubMed

15. Motzer RJ, Choueiri TK, Powles T, et al. Nivolumab + cabozantinib (NIVO+CABO) versus sunitinib (SUN) for advanced renal cell carcinoma (aRCC): outcomes by sarcomatoid histology and updated trial results with extended follow-up of CheckMate 9ER. J Clin Oncol 2021;39:308. DOI

16. Choueiri TK, Motzer RJ, Rini BI, et al. Updated efficacy results from the JAVELIN Renal 101 trial: first-line avelumab plus axitinib versus sunitinib in patients with advanced renal cell carcinoma. Ann Oncol 2020;31:1030-9. DOI PubMed

17. Rofi E, Del Re M, Arrigoni E, et al. Clinical pharmacology of monoclonal antibodies targeting PD-1 axis in urothelial cancers. Crit Rev Oncol Hematol 2020;154:102891. DOI PubMed

18. Porta C, Cosmai L, Rizzo M. Individualizing renal cell carcinoma treatment through biomarkers discovery in the era of immune checkpoint inhibitors: where do we stand? Curr Opin Urol 2021;31:236-41. DOI PubMed

19. Bracarda S, Sisani M, Marrocolo F, Hamzaj A, del Buono S, De Simone V. GOAL: an inverse toxicity-related algorithm for daily clinical practice decision making in advanced kidney cancer. Crit Rev Oncol Hematol 2014;89:386-93. DOI PubMed 